九州大学学術情報リポジトリ

Kyushu University Institutional Repository

\title{
Generation of virus-specific CD8+ T cells by vaccination with inactivated virus in the intestine of ginbuna crucian carp
}

Tajimi, Seisuke

Laboratory of Marine Biochemistry, Department of Bioscience and Biotechnology, Graduate School of Bioresource and Bioenvironmental Sciences, Kyushu University

Kondo, Masakazu

Department of Applied Aquabiology, National Fisheries University, Japan Fisheries Research and Education Agency

Nakanishi, Teruyuki

Department of Veterinary Medicine, Nihon University

Nagasawa, Takahi ro

Laboratory of Marine Biochemistry, Department of Bioscience and Biotechnology, Graduate School of Bioresource and Bioenvironmental Sciences, Kyushu University

他

http://hdl. handle. net/2324/2552964

出版情報: Developmental and Comparative Immunology. 93，pp.37-44，2019-04-01. Elsevier バージョン：

権利関係 : 


\section{Generation of virus-specific $\mathrm{CDB}^{+} \mathrm{T}$ cells by vaccination with inactivated virus in the intestine of ginbuna crucian carp}

Seisuke Tajimi ${ }^{\mathrm{a}}$, Masakazu Kondo ${ }^{\mathrm{b}}$, Teruyuki Nakanishic, Takahiro Nagasawa ${ }^{\mathrm{a}}$, Miki Nakao $^{\mathrm{a}}$, Tomonori Somamoto ${ }^{\mathrm{a} *}$.

${ }^{a}$ Laboratory of Marine Biochemistry, Department of Bioscience and Biotechnology, Graduate School of Bioresource and Bioenvironmental Sciences, Kyushu University, Fukuoka 819-0395, Japan.

${ }^{\mathrm{b}}$ Department of Applied Aquabiology, National Fisheries University, Japan Fisheries Research and Education Agency, Shimonoseki, Yamaguchi 759-6595, Japan

'Department of Veterinary Medicine, Nihon University, Fujisawa, Kanagawa 252-8510, Japan Phone: (81)-92-802-4792; Fax: (81)-92-802-4791 E-mail: somamoto@agr.kyushu-u.ac.jp 


\section{Abstract}

Although a previous study using ginbuna crucian carp suggested that cell-mediated immunity can be induced by the oral administration of inactivated viruses, which are exogenous antigens, there is no direct evidence that $\mathrm{CD}^{+}$cytotoxic $\mathrm{T}$ cells (CTLs) in teleost fish are generated by vaccination with exogenous antigens. In the present study, we investigated whether antigen-specific $\mathrm{CD}^{+}$CTLs in ginbuna crucian carp can be elicited by intestinal immunization with an exogenous antigen without any adjuvant. The IFN $\gamma$-1 and T-bet mRNA expressions were up-regulated in intestinal leukocytes following the administration of formalin-inactivated crucian hematopoietic necrosis virus (FICHNV), whereas the down-regulation of these genes was observed in kidney leukocytes. Furthermore, an increase in the percentage of proliferating $\mathrm{CD}^{+}$cells was detected in the posterior portion of the hindgut, suggesting that the virus-specific CTLs are locally generated in this site. In addition, cell-mediated cytotoxicity against CHNV-infected crucian carp 


\section{Introduction}

54 Cell-mediated immunity (CMI) executed by cytotoxic T-cells (CTLs) is an effective defense mechanism to suppress the propagation and spread of viruses in the host (Zinkernagel and Doherty, 1979). CTLs recognize a viral-antigen presented on MHC class I molecules and kill the virus-infected host cells. The viral peptides on the MHC class I molecules are intracellularly synthesized in the host cells. Therefore, inactivated viral vaccines, which are extracellular antigens, are not capable of efficiently eliciting CMI in hosts because they do not actively invade the cytosol of non-phagocytic host cells (Carbone and Bevan, 1990).

Fish are equipped with T-cell-mediated immunity similar to that present in mammals, and MHC class I-restricted cytotoxicity generated by CTLs is thought to serve an effective anti-viral function in teleost fish (Somamoto et al., 2014, Nakanishi et al., 2015, Munang'andu et al., 2015). Thus, similar to the mammalian system, the generation of viral antigen-specific CTLs in fish should also require stimulation with intracellular antigens. However, the expression profiles of T cell-related genes in several fish species suggest that immunization with exogenous antigens can induce virus-specific CTLs. Oral or bath vaccination with inactivated viruses induced the up-regulation of MHC class I and CD8 mRNA in teleost fishes (Ou-yang et al., 2012, Kai et al., 2014, Munang'andu et al., 2015), suggesting that vaccinations with inactivated viruses can also induce CMI. This finding implies that mucosal vaccination may be an effective method to generate CTLs in teleost fish.

The intestine is an important immune organ that protects against pathogen invasion and possesses gut-associated lymphoid tissue (GALT) that contains many intraepithelial 
cells comprise most of the IEL population found in the intestine, suggesting that the intestine is an important site for the development and activation of $\mathrm{T}$ cells in teleost fish (Rombout et al., 2011, Salinas 2015, Tafalla et al., 2016). Sato et al. (2005) reported that the direct administration of allogeneic cells into the intestine could induce systemic adaptive CMI in common carp. In studies on cell-mediated cytotoxicity (CMC) using ginbuna crucian carp, the oral administration of hapten-modified cells also elicited specific CMC activity (Sato and Okamoto 2008). Furthermore, the oral administration of formalin-inactivated crucian carp hematopoietic necrosis virus (CHNV) effectively prevented viral propagation in vivo and induced virus-specific CMC in ginbuna crucian carp (Sato and Okamoto 2010). These studies suggest that the fish intestine is an important site for generating CTLs. Based on the above findings, the following hypothesis can be proposed: the teleost fish immune system can easily generate virus-specific CTLs by immunizing the intestine with extracellular antigens. However, these studies failed to show any evidence that Th1-response and T-cell proliferation are induced by intestinal immunization. Therefore, the present study investigated the CTLs function, the transcriptional profile of T-cell-related molecules, the in vivo protective effect, and the in vivo proliferation of $\mathrm{CD}^{+}$cells following intestinal immunization with an inactivated virus. These results were then compared with the responses following intraperitoneal (i.p.) immunization.

\section{Materials and Methods}

2.1. Fish, cell lines, viruses, and formalin-inactivated CHNV

Two strains (OB1 and S3n) of isogeneic ginbuna crucian carp, Carassius auratus langsdorfii, were maintained at a temperature of $25^{\circ} \mathrm{C}$ and were fed daily with 
commercial food pellets. The fish were anesthetized with $25 \mathrm{mg} / \mathrm{L}$ quinaldine when injected or dissected. All experiments using fish were performed in accordance with the guidelines of the Animal Experiments Committee at Kyushu University. CFS (Carassius fin from Lake Suwa) cells from the S3n strain of ginbuna crucian carp were used as the syngeneic target cells and for propagation of the CHNV, as described by Somamoto et al. (2009, 2013). CHNV was inactivated with formalin by same procedure used in the previous study (Sato and Okamoto 2010).

\subsection{Immunization with FI-CHNV or live $C H N V$}

The intestinal immunization procedure used was that described in a previous study (Sato et al., 2005). Clonal ginbuna crucian carp (OB1 or S3n strain) were intubated with 0.5 $\mathrm{mL} / 100 \mathrm{~g}$ fish weight of a formalin-inactivated CHNV (FI-CHNV) solution (corresponding to $10^{8}$ TCID $50 / \mathrm{mL}$ in PBS) or a sublethal dose of live CHNV $\left(10^{6}\right.$ TCID $50 / 100 \mathrm{~g}$ weight) into their intestines from the anus via a polyethylene tube (0.5/1.0 mm inner/outer diameter). To prevent the solution from leaking back out, the part of the tube that corresponded to the length of the pelvic fin from the anus was gently inserted, and the solution was slowly administered into the hindgut. The control fish were intubated with PBS, instead of FI-CHNV or live CHNV. To compare intestinal immunization with systemic sensitization, i.p. immunization with FI-CHNV was performed using the same dose as that administered anally. The second immunization was performed one week after the primary sensitization using the same procedure.

\subsection{Gene expression analysis of T-cell-related genes}

124 The OB1 strain of ginbuna crucian carp, which weighed 21-24 g, underwent a gene 
expression assay using real-time PCR. Three fish in each group were sampled at 4, 8, and 12 days post-second sensitization with PBS and FI-CHNV or live CHNV. The trunk kidney and posterior intestine (the posterior one-third of the intestine) were excised from the fish. The trunk kidney was disaggregated by passing it through a 150-gauge mesh stainless steel sieve in RPMI-1640 (Nissui Pharmaceutical Co., Tokyo, Japan) supplemented with $1 \%$ heat-inactivated $\left(56^{\circ} \mathrm{C}\right.$ for $\left.30 \mathrm{~min}\right)$ fetal bovine serum (FBS: Biowest, France). The posterior intestines were homogenized using scissors and then disaggregated by being passed through a 150-gauge mesh stainless steel sieve in RPIM1640 containing 1\% FBS. The suspended cells were then applied to a discontinuous Percoll (GE healthcare) density gradient of 1.04 and $1.08 \mathrm{~g} / \mathrm{ml}$, and then centrifuged at $350 \times \mathrm{g}$ for $30 \mathrm{~min}$ at $4^{\circ} \mathrm{C}$. The cells present at the 1.04-1.08 interface were collected and washed twice with RPMI-1640. Total RNA was extracted from the isolated cells of the intestine and kidney using ISOGEN (Nippon gene, Tokyo, Japan), according to the manufacturer's protocol. First-strand cDNA was synthesized from $2 \mu \mathrm{g}$ of total RNA using the Moloney murine leukemia virus (M-MLV) reverse transcriptase (Invitrogen, Life Technologies, Carlsbad, CA, USA) with an oligo (dT) primer, according to the manufacturer's instructions. Primers used for the real-time PCR had been previously designed to amplify cDNA fragments encoding the following T-cell related genes: T-bet, GATA-3, IFN- $\gamma 1$, IFN- $\gamma 2$, IFN-rel2 (Yamasaki et al., 2014), IL-10, and T cell receptor- $\beta$ (TCR $\beta$ ) (Somamoto et al., 2009, 2015). EF-1 $\alpha$ served as an internal control for the normalization (Toda et al., 2011). The sequences or primer sets are indicated in the reference articles. Quantitative real-time PCR was performed in duplicate on a $\mathrm{M} \times 3000 \mathrm{P}$ System (Stratagene, La Jolla, CA) with $16 \mu \mathrm{L}$ reaction mixtures containing $2 \mu \mathrm{l}$ of template cDNA, $0.5 \mu \mathrm{M}$ primers, and other reagent components from the Fast Start DNA Master SYBR_Green (Roche Applied Science, Mannheim, Germany). Thermal cycling was performed using a two-step thermal cycling mode composed of an initial denaturation for $10 \mathrm{~min}$ at $95^{\circ} \mathrm{C}$, followed by 40 cycles of $15 \mathrm{sec}$ at $95^{\circ} \mathrm{C}$ and $60 \mathrm{sec}$ at $61^{\circ} \mathrm{C}(\mathrm{EF} 1 \alpha$, 
IFN $\gamma$-1, IFN $\gamma$-2, IFN $\gamma$-rel2, GATA-3, T-bet, and TCR $\beta$ ) or 40 sec at $60^{\circ} \mathrm{C}$ (IL-10). For all the quantitative real-time PCR assays, melting curve analyses were performed and single specific melting peaks were observed, which indicated amplification specificity. The relative quantitative value of each gene was calculated according to the standard curve from a serial dilution of a reference cDNA in the same plate and normalized with the level of EF1 $\alpha$. Data from the three individual fish are shown as the mean fold change in mRNA expression relative to the PBS-administered fish.

\subsection{Detection of proliferating cell nucleic antigens (PCNAs) in CD8 $\alpha^{+}$cells by flow} cytometry (FCM)

To determine whether CD8 $\alpha^{+}$cells proliferated in the kidney and intestine in vivo after FI-CHNV-sensitization, the PCNA protein, which is expressed in cells in the later stages of the $\mathrm{G} 1$ and S-phases, was detected in CD8 $\alpha^{+}$cells by FCM. The OB1 strain of ginbuna crucian carp, weighing 4.8-7.9 g, was used in this experiment. Intestinal or i.p. immunization with FI-CHNV was followed according to the method described above. To reveal a more detailed site for generating virus-specific CTLs in the posterior intestine, the posterior intestine was divided into two parts as shown in Fig. S1. The leukocytes from the tissues were collected at 2 and 4 dpi from the immunized or control fish (four fish in each group). The number of collected cells was counted on a hemocytometer. The collected cells from the tissues were incubated in a 1:10,000 dilution of rat anti-ginbuna CD8 $\alpha$ mAbs (mouse ascites) for 40 min on ice (Toda et al., 2011). The cells were then washed twice with OPTI-MEM (Invitrogen, Carlsbad, CA) and incubated for $30 \mathrm{~min}$ at $4^{\circ} \mathrm{C}$ with a 1:100 dilution of anti-rat donkey IgG $(\mathrm{H}+\mathrm{L})$ antibodies conjugated with Alexa Fluor 488 (Invitrogen, Carlsbad, CA). The control samples were stained with just the secondary antibody (no primary antibody). After two washes with the medium, the cells 
suspended in OPTI-MEM supplemented with 1\% FBS (OPTI-1) were mixed with the same volume of 4\% paraformaldehyde (PFA) PBS solution (Nacalai Tesque Inc., Kyoto, Japan) (final conc. 2\% PFA) and were incubated for $20 \mathrm{~min}$ at room temperature. The fixed cells were washed three times and suspended in PBS containing $0.2 \%$ saponin (Nacalai Tesque Inc., Kyoto, Japan) and 3\% bovine serum albumin (BSA) (PBS-SapoBSA); they were then incubated for one hour at room temperature. The membranepermeabilized cells were incubated with a 1:100 dilution of anti-PCNA mouse mAbs (PC10; Santa Cruz Biotechnology), which have been shown to react with cells from various species including Drosophila, zebrafish, and ginbuna crucian carp (Nakanishi and Ototake 1999), or the same concentration of normal mouse IgG (IgG2a) as a control, for $40 \mathrm{~min}$ at room temperature. After being washed twice with OPTI-1, the cells were resuspended in PBS-Sapo-BSA and incubated with a 1:100 dilution of anti-mouse IgG goat antibodies conjugated with PerCP/Cy5.5 (Biolegend, San Diego, CA). The labeled cells were washed twice and suspended in OPTI-1. The fluorescent stained cells were analyzed with an Attune NxT Flow Cytometer (Thermo Fisher Scientific, Waltham, MA, USA). The region including $C D 8 \alpha^{+}$cells was gated and classified as the lymphocyte fraction. Alexa Fluor 488 or PerCP/Cy5.5 fluorescence was detected in the BL1 channel using a 530/30 nm bandpass filter or the BL3 channel using a 695/40 nm bandpass filter with $488 \mathrm{~nm}$ excitation, respectively.

\subsection{CMC against CHNV-infected syngeneic cells}

The S3n strain of ginbuna crucian carp, weighing 35-72 g, was used in the cytotoxic assays. The fish were sensitized twice via anal administration or i.p. injection with FICHNV as described above. Blood was collected from the fish at 4 and 8 dpi (four fish per 
sample). Peripheral blood leukocytes (PBLs) were used as effector cells to evaluate the systemic response in the CMC assay because the spontaneous release of LDH from PBLs was low. PBLs were isolated using a Percoll gradient method as previously described (Somamoto et al., 2015). The PBLs were then suspended in DMEM/F-12 medium (Invitrogen, Carlsbad, CA) without phenol red and with 1\% heat-inactivated FBS (DMEM/F12-1); they were then used as effector cells for a cytotoxicity assay. The cytotoxicity was assayed as previously described (Somamoto et al., 2015). In brief, CFS cells were used as syngeneic target cells, seeded in 96-well, flat-bottom microtiter plates (Nunc, Roskilde, Denmark) at a concentration of $10^{4}$ cells/well, and allowed to settle for $6 \mathrm{~h}$. Half of the wells with the target cells were infected with $\mathrm{CHNV}$ at $25^{\circ} \mathrm{C}$ for $3-4 \mathrm{~h}$ $(\mathrm{MOI}=10)$. Effector: target ratios were adjusted to 80:1 and 40:1. At least duplicate wells were analyzed for each test. The maximum amount of lactate dehydrogenase (LDH) released by the infected and uninfected targets was determined by damaging the cells with 1\% Triton X-100 (high control). Spontaneous LDH release from the effector and target cells was determined with untreated cells that were cultured with only medium (low control). Cytotoxic activity against the infected and uninfected targets was detected by LDH release using a Cytotoxicity Detection Kit-LDH (Takara Bio Inc., Shiga, Japan), according to the manufacturer's protocol. The absorbance of plates containing the supernatants from the test wells and the substrate mix were determined with an ELISA reader at $490 \mathrm{~nm}$. The value was subtracted from the background (only medium or medium + Triton X-100). There was no significant difference in spontaneous release between the uninfected and infected target cells.

The percentage of specific cytotoxicity was calculated using the following formula: Cytotoxicity $(\%)=\mathrm{A}-$ low control/high control - low control $\times 100$ (A: [effector - target 
cell mix] - [effector cell control]).

CD8 $\alpha^{+}$and $C D 8 \alpha^{-}$cells were separated from PBLs using magnetic cell separation as previously described (Somamoto et al., 2013). The cytotoxic activity of CD8 $\alpha^{+}$and CD8 $\alpha^{-}$cells from fish sampled at 8 dpi was measured as described above. Effector: target ratios were adjusted to 20:1 and 10:1.

\subsection{Virus titers in organs of vaccinated fish after CHNV infection}

The organ virus titers were measured following the protocols of previous studies

233 (Somamoto et al., 2013; Sato and Okamoto 2010). Ginbuna crucian carp (OB1 strain), weighing 34-58 g, were used in this experiment. The fish (four fish per group) were anally administered FI-CHNV or PBS into the hindgut and were intraperitoneally injected with FI-CHNV as described above. At $14 \mathrm{dpi}$, the sensitized fish were intraperitoneally immunized with $\mathrm{CHNV}$ at $10^{6}$ TCID $50 / 50$ g body weight. The spleen and trunk kidney were collected at 3 dpi and placed in MEM (Nissui Pharmaceutical Co., Tokyo, Japan) (1:10, weight: volume) containing $10 \%$ heat-inactivated FBS, homogenized, and then centrifuged at $2000 \times \mathrm{g}$ for $20 \mathrm{~min}$. The supernatants were collected, passed through a $0.45-\mu \mathrm{m}$ membrane filter, and stored at $-80^{\circ} \mathrm{C}$ until required. The virus titers were determined according to a TCID 50 endpoint titration in CFS cells incubated for 21 days at $25^{\circ} \mathrm{C}$. The results were expressed as the TCID 50 per gram of organ.

\subsection{Statistical analysis}

The results were expressed as the mean \pm standard deviation. Statistical differences were assessed for more than two groups using an analysis of variance (one-way ANOVA) followed by Tukey's comparison test or analyzed by student's $t$-test for comparison 
between two groups. The significance level was set at 0.05 .

\section{Results}

\subsection{Expression of T-cell-related molecules after FI-CHNV and live CHNV sensitization} The expressions of TCR $\beta$ as well as the transcription factors GATA-3 and T-bet are shown in Fig. 1A. The intestinal immunization with FI-CHNV induced up-regulations of T-bet in intestinal leukocytes at 4 and 8 dpi, whereas the expression in kidney leukocytes significantly decreased at $4 \mathrm{dpi}$ and showed no up-regulation at 8 and $12 \mathrm{dpi}$. On most sampling days, the expression level of TCR $\beta$ mRNA in the kidney and intestine was significantly higher than that in the control fish, although there was no difference in the kidney leukocytes sampled at 12 dpi. Conversely, there was no significant difference in GATA-3 mRNA expression at any time point. The expression profiles induced by i.p. immunization were largely similar to those induced by intestinal immunization; however, the expression of TCR $\beta$ mRNA was not enhanced by immunization. The expression profiles of cytokine expression in the FI-CHNV-immunized fish are shown in Fig. 1B. Intestinal immunization significantly enhanced IFN $\gamma 1$ expression in intestinal leukocytes sampled at 4 and $8 \mathrm{dpi}$, whereas the expression in kidney leukocytes was significantly lower than that in the control fish. Systemic immunization also induced the enhancement in intestinal leukocytes, but not in kidney leukocytes. Although significant up-regulation of IFN- $\gamma 2$ was not observed in the intestinal immunized fish, the expression of IFN $\gamma$-rel mRNA was increased in intestinal leukocytes at 8 and 12 dpi. In both organs, the expression profiles of IL-10 mRNA were enhanced following the immunizations. 
similar pattern as those in the fish immunized with FI-CHNV via the intestine, whereas the expression of IFN $\gamma 1$ in the intestine was not enhanced at any time point by the immunization.

\subsection{Proliferation of $\mathrm{CD}^{+}$cells in the intestine and kidney by FI-CHNV-sensitization} Whether virus-specific $\mathrm{CD}^{+}$cells were generated in the intestine or kidney after FICHNV administration was investigated by employing an FCM analysis using anti-PCNA antibodies (Fig. 2). There is no significant difference of the total number of collected cells among each group. The region including $C D 8 \alpha^{+}$cells was gated and regarded as the lymphocyte fraction. Double positive (DP) cells, which express both CD8 $\alpha$ and PCNA, were detected in the kidney and intestine (Fig. 2A). In the posterior part of the hindgut from fish sampled at 2 dpi (Intestine 2), the percentage of DP cells in the intestinally immunized fish was significantly higher than that in either the PBS-administrated fish or the intraperitoneally immunized fish. Conversely, there were no significant differences in DP cell composition in the kidney or the anterior part of the hindgut among the treated groups (Intestine 1). Although the normal PCNA protein expression level was higher in the kidney than in the intestine, no significant differences in DP cell percentages among the treated groups were observed for either organ. In all groups that were sampled at 4 dpi, the percentages of DP cells did not increase as a result of FI-CHNV-sensitization. The $\mathrm{CD}^{+}$cell percentages in the intestines of FI-CHNV-immunized fish did not increase or were significantly lower than those of PBS-administered fish at some sampling times. 
To determine whether CMI can be induced as a result of immunization with FI-CHNV, the cytotoxicity of PBL against CHNV-infected cells was analyzed using the syngeneic target cell line (Fig. 3). At 8 dpi, the unsorted effector cells from the fish immunized with FI-CHNV efficiently killed CHNV-infected syngeneic cells, but not uninfected cells ( $\mathrm{p}<$ 0.05). Meanwhile, the effector cells from PBS-administered fish did not show significant activity against CHNV-infected cells (Fig. 3A). The effector cells that were collected at 4 dpi did not significantly kill the infected cells. The cytotoxicity of $\mathrm{CD}^{+}$cells against CHNV-infected cells was significantly higher than that against uninfected cells; conversely, CD8 ${ }^{-}$cells showed the same level of activity (Fig. 3B). The activities at lower effector/target ratios were reduced in all assays (data not shown). These results indicate that both intestinal and systemic immunization with FI-CHNV can induce CTL activity against virus-infected cells.

\subsection{Protective effect of FI-CHNV vaccination}

The vaccination effects of intestinal and i.p. FI-CHNV administration were determined by evaluating the viral load of the spleen and kidney from the fish that were infected with CHNV after the vaccination (Fig. 4). In both the kidney and spleen, the viral titer of the fish that were intestinally immunized with FI-CHNV was significantly lower than that of the fish that were intestinally administered PBS; however, no significant reduction was observed in the kidney of the i.p. FI-CHNV-immunized fish. Furthermore, there was no significant difference in the viral load between both routes of FI-CHNV immunization in the spleen and kidney of the fish. These results suggest that the administration of the inactivated virus can efficiently prevent viral replication in vivo. 


\section{Discussion}

CMC by CD8 $\alpha^{+}$CTLs is thought to be an effective mechanism for controlling viral infection in teleost fish and in mammals (Somamoto et al., 2014, Zinkernagel and Doherty 1979). MHC class I and antigen-presentation and -processing related molecules have been identified in many fish species (Grimholt 2016, Wilson 2017), and functional and structural analyses suggest that the antigen-presentation mechanism in teleosts is similar to that in mammals (Sever et al 2014, Chen et al., 2010, 2017). Thus, the induction of viral-antigen-specific $\mathrm{CD}^{+}$CTLs in fish is believed to require stimulation with endogenous antigens in MHC class I antigen processing machinery. The crosspresentation is essential for the presentation of exogenous antigens on MHC class I molecules and the initiation of $\mathrm{CD}^{+}$T-cell responses in mammals (Joffre et al., 2012). Recent studies suggest that teleost DC-like cells possess cross-presentation ability (Granja et al., 2015, Soleto et al., 2018). The present study has shown that immunization with exogenous antigens can induce a Th1 response and CMC by CD8 ${ }^{+}$cells, implying organs or the blood.

T-bet is a transcription factor that regulates Th1 cell differentiation and controls the expression of IFN- $\gamma$ in mammals (Szabo et al., 2000, Sullivan et al., 2003), and its expression is skewed by Th-1. Several studies have indicated that the expression level of the T-bet gene is correlated to Th-1 responses in teleost fish (Munang'andu et al., 2013, 
Yamasaki et al., 2014, 2015, Yang et al., 2017). An interesting observation in the present study is that the enhancement of T-bet expression in FI-CHNV-immunized fish was detected in the intestine, but not in the kidney. The IFN- $\gamma$ mRNA expression profile agrees with this observation, although higher IL-10 mRNA expression levels show conflicting results. The expression of GATA-3, which is essential for the differentiation of mature naive $\mathrm{CD}^{+}$T-cells to Th-2 cells, was not elevated in the kidney or intestine by the stimulation. Comprehensively, the expression analysis of T-cell related genes shows that the Th1 response is induced by intestinal and systemic administration of the inactivated virus. Furthermore, we have demonstrated that the posterior part of the intestine is the site of $\mathrm{CD}^{+}$cell generation by immunization with the inactivated virus. In IPNV-sensitized rainbow trout, $\mathrm{T}$ cells mobilized to the mid/hindgut region and pyloric caeca, but not the foregut region (Ballesteros et al., 2014). Although it cannot simply correspond to the regions in trout and crucian carp, which have physiologically different digestive tracts, this finding and our results suggest that the posterior region of the digestive tract is an important T cell mobilization site in teleosts. There are various phenotypes of CD8 ${ }^{+}$IELs, including TCR $\alpha \beta^{+} \mathrm{T}$ cells, TCR $\gamma \delta^{+} \mathrm{T}$ cells, and natural killer T (NKT) cells in mammals (Bannai et al., 2003, Konjar et al., 2017), suggesting that teleost IELs are also classified into various phenotypes. Thus, further studies should identify the population of $\mathrm{CD}^{+}$ IELs present in ginbuan crucian carp.

CMC against CHNV-infected syngeneic cells was induced by immunization with FICHNV. This result is consistent with the report that CMC activity was induced by oral immunization with FI-CHNV (Sato and Okamoto 2010). Furthermore, the present study has determined that CD8 $\alpha^{+}$cells are effectors against virus-infected cells in FI-CHNVimmunized fish. Previous studies have shown that $\mathrm{CD} 8 \alpha^{+}\left(2 \mathrm{C} 3 \mathrm{mAb} \mathrm{b}^{+}\right)$lymphocytes 
strongly express TCR $\beta$, lck, CD3e, and ZAP70 mRNA (Toda et al., 2011, Somamoto et al., 2013, Miyazawa et al., 2018) as well as ZAP70 and CD3 $\varepsilon$ proteins (Miyazawa et al., 2018). In addition, a previous study reported that CHNV-sensitized effector cells, which express TCR $\beta$ and CD8 $\alpha$ mRNA, possess CMC against CHNV-infected syngeneic cells but not against infected allogeneic cells, implying that they recognize virus-infected targets in an MHC class I restriction manner (Somamoto et al., 2002, 2009). These findings suggest that CD8 $\alpha^{+}$CTLs are the dominant effector cells in the present study, although further identification of the effector cells would be required. Although CD8 $\alpha^{-}$ cells also significantly killed CHNV-infected cells in this study, the effector cells except for CTLs contributed to CMC against CHNV-infected cells, as shown in the previous study (Somamoto et al., 2013). We suggest that monocytes and/or NK-like cells were activated by Th-1 cytokines such as IFN $\gamma$ and thus play an important role in the clearance of viral infections. In fact, a large number of CD8- cells were proliferated by the immunization with FI-CHNV, suggesting that leukocytes other than CTLs also contribute to controlling CHNV-infection. Intestinal immunization with FI-CHNV provided significant prevention against CHNV-replication in the kidney and spleen, suggesting that CMC by CTLs and other effecter cells contributed to this protection. Evidence at the cellular and biological levels indicates that vaccination with extracellular antigens can induce efficient CMC, thus demonstrating its availability as a useful viral vaccine for fish. Our hypothesis was that mucosal vaccination can induce a more efficient cell-mediated immune response than systemic vaccination because several studies have suggested that Th1 responses were induced by bath or oral vaccinations with inactivated virus (Sato and 
cells and protection against CHNV-infection, whereas the effect was slightly lower than that by intestinal immunization. Interestingly, both systemic and intestinal immunization generated viral antigen reactive $\mathrm{CD}^{+}$cells and enhanced Th1-related genes in the intestine, but not in the kidney. This fact implies that the posterior part of the intestine possesses an important site for cross-presentation, which can present exogenous antigens on MHC class I, by dendritic cells (DCs). Teleost DC-like cells have recently been identified in rainbow trout and are inferred to possess cross-presentation ability (Granja et al., 2015, Soleto et al., 2018). The trout DC-like cells were found in the gills and skin and showed different phenotypic and functional characteristics, suggesting the existence of distinct DC subsets in the mucosal tissues. Therefore, mucosal tissues may be important sites for developing DCs in teleost fish, which indicates that the mucosal DCs play a major role in generating antigen-specific $\mathrm{CD}^{+}$cells. According to the findings from the present study, we hypothesize that the intestinal resident DCs ingested FI-CHNV, which was directly exposed to or circulated into the intestine, by endocytosis, processed it via proteasomes, and presented it on MHC class I molecules to CD8 ${ }^{+}$cells. The cross presentation frequently occurs in mucosal tissues such as the intestine. Further studies on intestinal DCs would provide a better understanding of the mechanisms of how inactivated vaccines induce virus-specific CTLs.

\section{Acknowledgments}

This work was supported in part by the Japan Society for the Promotion of Science KAKENHI Grant Number 26450286, 17K07939, 16H04984. The authors would like to thank Enago (www.enago.jp) for the English language review. 


\section{References}

Bannai, M., Kawamura, T., Naito, T., Kameyama, H., Abe, T., Kawamura, H., Tsukada, C., Watanabe, H., Hatakeyama, K., Hamada, H., Nishiyama, Y., Ishikawa, H., Takeda, K., Okumura, K., Taniguchi, M., Abo, T., 2001. Abundance of unconventional CD8(+) natural killer T cells in the large intestine. Eur. J. Immunol. 31, 3361-9.

Ballesteros, N.A., Rodríguez Saint-Jean, S., Pérez-Prieto, S.I., Aquilino, C., Tafalla, C., 2014. Modulation of genes related to the recruitment of immune cells in the digestive tract of trout experimentally infected with infectious pancreatic necrosis virus (IPNV) or orally vaccinated. Dev. Comp. Immunol. 44, 195-205.

Brandtzaeg, P., Pabst, R., 2004. Let’s go mucosal: communication on slippery ground. Trends Immunol. 25, 570-577.

Carbone, B.F.R., Bevan, M.J., 1990. Class I-restricted processing and presentation of exogenous cell-associated antigen in vivo. Rockefeller Univ. Press 171, 377-387. https://doi.org/2137512

Chen, W., Jia, Z., Zhang, T., Zhang, N., Lin, C., Gao, F., Wang, L., Li, X., Jiang, Y., Li, X., Gao, G.F., Xia, C., 2010. MHC class I presentation and regulation by IFN in bony fish determined by molecular analysis of the class I locus in grass carp. J. Immunol. 185, 2209-2221.

Chen, Z., Zhang, N., Qi, J., Chen, R., Dijkstra, J.M., Li, X., Wang, Z., Wang, J., Wu, Y., Xia, C., 2017. The structure of the MHC class I molecule of bony fishes provides insights into the conserved nature of the antigen-presenting system. J. Immunol. 199, 3668-3678.

Granja, A.G., Leal, E., Pignatelli, J., Castro, R., Abós, B., Kato, G., Fischer, U., Tafalla, C., 2015. Identification of teleost skin CD8 $\alpha^{+}$dendritic-like cells, representing a potential common ancestor for mammalian cross-presenting dendritic ells. J. Immunol. 195, 1825-37.

Grimholt, U., 2016. MHC and evolution in teleosts. Biology (Basel). 5, 6. 
Joffre, O.P., Segura, E., Savina, A., Amigorena, S., 2012. Cross-presentation by dendritic cells. Nat. Rev. Immunol. 12, 557-569.

Kai, Y.-H., Wu, Y.-C., Chi, S.-C., 2014. Immune gene expressions in grouper larvae (Epinephelus coioides) induced by bath and oral vaccinations with inactivated betanodavirus. Fish Shellfish Immunol. 40, 563-9.

Miyazawa, R., Murata, N., Matsuura, Y., Shibasaki, Y., Yabu, T., Nakanishi, T., 2018. Peculiar Expression of CD3-Epsilon in Kidney of Ginbuna Crucian Carp. Front. Immunol. 9, 1321.

Konjar, Š., Ferreira, C., Blankenhaus, B., Veldhoen, M., 2017. Intestinal Barrier Interactions with Specialized CD8 T Cells. Front. Immunol. 8, 1281.

Munang’andu, H.M., Fredriksen, B.N., Mutoloki, S., Dalmo, R.A., Evensen, Ø., 2013. The kinetics of $\mathrm{CD}^{+}$and $\mathrm{CD}^{+} \mathrm{T}$-cell gene expression correlate with protection in Atlantic salmon (Salmo salar L) vaccinated against infectious pancreatic necrosis. Vaccine 31, 1956-1963.

Munang’andu, H.M., Mutoloki, S., Evensen, Ø., 2015. A Review of the immunological mechanisms following mucosal vaccination of Finfish. Front. Immunol. 6, 427.

Nakanishi, T., Ototake, M.,1999. The graft-versus-host reaction (GVHR) in the ginbuna crucian carp, Carassius auratus langsdorfii. Dev. Comp. Immunol. 23, 15-26.

Nakanishi, T., Shibasaki, Y., Matsuura, Y., 2015. T cells in fish. Biology (Basel). 4, 640-663.

Ou-yang, Z., Wang, P., Huang, X., Cai, J., Huang, Y., Wei, S., Ji, H., Wei, J., Zhou, Y., Qin, Q., 2012. Immunogenicity and protective effects of inactivated Singapore grouper iridovirus (SGIV) vaccines in orange-spotted grouper, Epinephelus coioides. Dev. Comp. Immunol. 38, 254-61.

Rombout, J.H.W.M., Abelli, L., Picchietti, S., Scapigliati, G., Kiron, V., 2011. Teleost intestinal immunology. Fish Shellfish Immunol. 31, 616-626.

Salinas, I., 2015. The mucosal immune system of teleost fish. Biology (Basel). 4, 52539. 
Sato, A., Okamoto, N., 2008. Characterization of the cell-mediated cytotoxic responses of isogeneic ginbuna crucian carp induced by oral immunisation with haptenmodified cellular antigens. Fish Shellfish Immunol. 24, 684-92.

Sato, A., Okamoto, N., 2010. Induction of virus-specific cell-mediated cytotoxic responses of isogeneic ginbuna crucian carp, after oral immunization with inactivated virus. Fish Shellfish Immunol. 29, 414-21.

Sato, A., Somamoto, T., Yokooka, H., Okamoto, N., 2005. Systemic priming of alloreactive cytotoxic cells in carp, following anal administration of allogeneic cell antigens. Fish Shellfish Immunol. 19, 43-52.

Sever, L., Vo, N.T.K., Lumsden, J., Bols, N.C., Dixon, B., 2014. Induction of rainbow trout MH class I and accessory proteins by viral haemorrhagic septicaemia virus. Mol. Immunol. 59, 154-62.

Soleto I, Fischer U, Tafalla C, Granja AG. 2018. Identification of a potential common ancestor for mammalian cross-presenting dendritic cells in teleost respiratory surfaces. Front Immunol. 25;9:59.

Somamoto, T., Koppang, E.O., Fischer, U., 2014. Antiviral functions of CD8 cytotoxic T cells in teleost fish. Dev. Comp. Immunol. 2014, 43, 197-204.

Somamoto, T., Miura, Y., Nakanishi, T., Nakao, M., 2015. Local and systemic adaptive immune responses toward viral infection via gills in ginbuna crucian carp. Dev. Comp. Immunol. 52, 81-87.

Somamoto, T., Nakanishi, T., Nakao, M., 2013. Identification of anti-viral cytotoxic effector cells in the ginbuna crucian carp, carassius auratus langsdorfii. Dev. Comp. Immunol. 39, 370-377.

Somamoto, T., Okamoto, N., Nakanishi, T., Ototake, M., Nakao, M., 2009. In vitro generation of viral-antigen dependent cytotoxic T-cells from ginbuna crucian carp, carassius auratus langsdorfii. Virology 389, 26-33.

Sullivan, B.M., Juedes, A., Szabo, S.J., von Herrath, M., Glimcher, L.H., 2003. Antigen-driven effector CD8 T cell function regulated by T-bet. Proc. Natl. Acad. Sci. 100, 15818-15823. 
Szabo, S.J., Kim, S.T., Costa, G.L., Zhang, X., Fathman, C.G., Glimcher, L.H., 2000. A novel transcription factor, T-bet, directs Th1 lineage commitment. Cell 100, 655669.

Tafalla, C., Leal, E., Yamaguchi, T., Fischer, U., 2016. T cell immunity in the teleost digestive tract. Dev. Comp. Immunol. 64, 167-77.

Toda H, Saito Y, Koike T, Takizawa F, Araki K, Yabu T, Somamoto T, Suetake H, Suzuki Y, Ototake M, Moritomo T, Nakanishi T. 2011. Conservation of characteristics and functions of CD4 positive lymphocytes in a teleost fish. Dev Comp Immunol. 2011. 35, 650-60.

Wilson, A.B., 2017. MHC and adaptive immunity in teleost fishes. Immunogenetics 69, 521-528.

Yamasaki, M., Araki, K., Maruyoshi, K., Matsumoto, M., Nakayasu, C., Moritomo, T., Nakanishi, T., Yamamoto, A., 2015. Comparative analysis of adaptive immune response after vaccine trials using live attenuated and formalin-killed cells of Edwardsiella tarda in ginbuna crucian carp (Carassius auratus langsdorfii). Fish Shellfish Immunol. 45, 437-442.

Yamasaki, M., Araki, K., Nakanishi, T., Nakayasu, C., Yamamoto, A., 2014. Role of CD4+ and CD8 $\alpha+T$ cells in protective immunity against Edwardsiella tarda infection of ginbuna crucian carp, Carassius auratus langsdorfii. Fish Shellfish Immunol. 36, 299-304.

Yang, S., Li, Q., Mu, Y., Ao, J., Chen, X., 2017. Functional activities of interferon gamma in large yellow croaker Larimichthys crocea. Fish Shellfish Immunol. 70, 545-552.

Zinkernagel, R.M., Doherty, P.C., 1979. MHC-restricted cytotoxic T cells: studies on the biological role of polymorphic major transplantation antigens determining $\mathrm{T}$ cell restriction-specificity, function, and responsiveness. Adv. Immunol. 27, 51177.

\section{Figure legends}


Figure 1. Quantitative expression profiles of T-cell-related genes (TCR $\beta$, T-bet, and GATA-3) (A) and cytokine genes (IFN $\gamma 1$, IFN $\gamma 2$, IFN $\gamma$-rel2, and IL-10) (B) in kidney (left graph) and intestine (right graph) cells after CHNV-sensitization. Data from the three individual fish are shown as the mean fold change in mRNA expression relative to the PBS-administered fish (white bars). The fish were immunized with FI-CHNV (black bars) or live CHNV (hatched bars) via the intestine and with FI-CHNV via i.p. injection $0.05)$.

540

Figure 2. Detection of PCNA ${ }^{+}$and $\mathrm{CD} 8 \alpha^{+}$cells by FCM in the kidney and intestines of FI-CHNV-immunized fish. (A) Two color histograms of cells expressing CD8 $\alpha$ (BL1) and PCNA (BL3). Cells that were sensitized by intestinal (CHNV-Int) and intraperitoneal (CHNV-Ip) immunization with FI-CHNV or intestinal administration with PBS were analyzed by FCM. The cells were collected from the kidney, the anterior portion of the hindgut (intestine 1), and the posterior portion of the hindgut (intestine 2). Four individual samples were independently analyzed, and representative data are shown. The numbers indicate the percentages of positive cells within each region. The control samples were stained with isotype control antibodies (BL3) or with only secondary antibodies (no primary antibody) (BL1). (B) Percentages of CD8 $\alpha$-positive and double positive (DP) cells. The results are presented as the means of four individual fish, and the error bars indicate SD. The asterisks indicate significant differences between each group ( $* \mathrm{P}<0.05)$.

Figure 3. Cell-mediated cytotoxic activity of unsorted leukocytes (A) or CD8 $\alpha^{+} / \mathrm{CD} 8 \alpha^{-}$ 
cells (B) against CHNV-infected syngeneic cells. Unsorted leukocytes that were sensitized by intestinal (Int) and intraperitoneal (Ip) immunization with FI-CHNV or intestinal administration with PBS were used as effector cells. CD8 $\alpha^{+}$and CD8 $\alpha^{-}$cells that were sensitized by intestinal immunization with FI-CHNV were used as effector cells. The unsorted cells or the $\mathrm{CD} 8 \alpha^{+} / \mathrm{CD} 8 \alpha^{-}$cells were incubated with target cells at an effector:target ratio of $80: 1$ or 20:1, respectively. The unsorted cells were collected from fish sampled at 4 (a) and 8 (b) dpi. CD8 $\alpha^{+} / \mathrm{CD} 8 \alpha^{-}$cells were isolated from fish sampled at $8 \mathrm{dpi}$. The results are presented as the means of four individual fish, and the error bars indicate the SD. The black and white bars indicate the activities against the infected and uninfected cells, respectively. The asterisks indicate significant differences from the activity against uninfected targets $(* \mathrm{P}<0.05)$.

566

567

Figure 4. Viral loads of kidneys and spleens from vaccinated fish. The vaccinated fish were anally or intraperitoneally immunized with FI-CHNV. The results are presented as the means of four fish, and the error bars indicate the standard deviations (SDs). Means with different letters indicate significant differences $(* \mathrm{P}<0.05)$. 